\title{
BIFURCACIONES EN MODELOS SOBRE IDENTIFICACIÓN DE PATRONES
}

\section{BIFURCATIONS IN MODELS FOR PATTERN IDENTIFICATION}

\author{
Mauro Montealegre Cárdenas, \\ Jasmidt Vera Cuenca, Gustavo Londoño Betancourth*
}

\begin{abstract}
Resumen
Existe una relación muy importante entre los estudios de los fenómenos interdisciplinarios y la metodología de identificación de patrones, porque esta ayuda a entender mejor el mismo fen'omeno. Por eso presentamos en este articulo una descripción analítica de los conceptos básicos de la teoría de reconocimiento de patrones, ilustrando y describiendo las bifurcaciones de modelos genéricos que han surgido en los últimos años en diversos campos del conocimiento.
\end{abstract}

Palabras claves: Identificación de patrones, linealización homogenea, ecuación de amplitud, ecuaciones de fases, bifurcaciones.

\section{Abstract}

There is a very important relationship between the studies of interdisciplinary phenomena and research methodology patterns, because this helps to better understand the same phenomena. For this reason we present in this article an analytical description of the basic concepts of the theory of pattern recognition, illustrating and describing the Bifurcations of generic models that have emerged in recent years in various fields of knowledge.

Key words: identification of patterns, homogeneous linearization, amplitude equation, phase equation, bifurcations.

\section{Introducción}

Este artículo es un resultado del proyecto de investigación denominado "Bifurcaciones en modelos matemáticos de identificaci' on de patrones y aplicaciones", y en el presentamos una metodología sobre la bifurcación de identificación de patrones, como resultado de una indagación transversal en la bibliografía relacionada al final de este trabajo, y tambíen explorando aplicaciones que interesan a la comunidad científica interdisciplinaria; en particular los campos de la hidrología, dinámica climática, semiconductores, modelos biológicos, entre otros. De otro lado, estos estudios son una oportunidad para interactuar con las teorías matemáticas que involucran la evolución de sistemas dinámicos, el an'alisis funcional y la geometría de dichos patrones.

Con los ejemplos de la segunda sección ilustramos esta metodología, en particular, profundizamos en la comprensión del modelo denominado Kuramoto- Sivashinsky, el cual aparece en diversos contextos para explicar estructuras turbulentas incluyendo la aparición del caos espacio-temporal.

En la tercera sección de este artículo relacionamos los componentes básicos que ayudan a la identificación de patrones como: la ecuación de amplitud, ecuacíon de fases y una metodología para conocer sus ecuaciones de bifurcaciones, según existan o no, simétricas u oscilaciones.

* Investigadores Grupo de Investigación DINUSCO. Email: mmontealegre.cardenas@gmail.com 


\section{Modelos Básicos sobre Identificación de Patrones}

i) Hacia una teoría computacional de la morfogénesis

Las capacidades computacionales de la tortuga puede ser reducido si la tortuga se le permite recibir señales de su entorno. En el ejemplo mostrado en la Figura 1, se supone que el medio ambiente es una cuña limitada por dos líneas que se cortan. La tortuga se mueve verticalmente hasta que se encuentra con cualquiera de estas líneas de guía, momento en el cual se realiza un cambio de sentido.
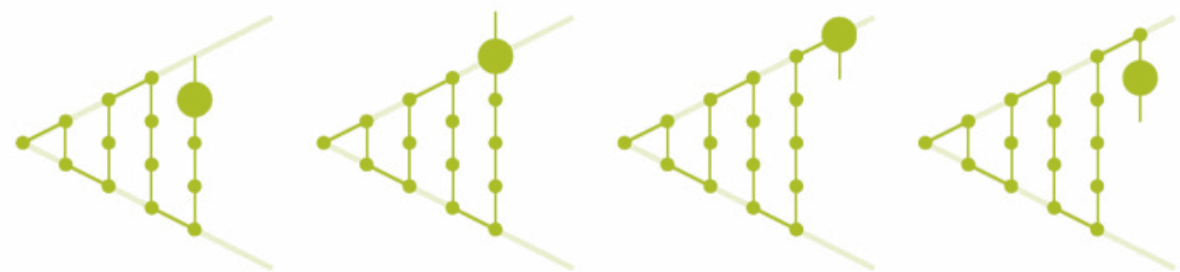

Figura 1: Zig-zag creado por una tortuga recibiendo señales desde el entorno

Este proceso puede estar relacionado con la formación de patrones en zig-zag por minadores de la hoja, larvas de insectos que viven y se alimentan dentro de una hoja. En el proceso de alimentación, crean túneles que son visibles como senderos finos en el limbo de la hoja. Un ejemplo del patrón resultante se muestra en la Figura 2. Tiene un carácter que recuerda serpenteo de una curva en zig-zag, y está limitado por las venas de las hojas principales, que la larva no cruzan fácilmente.

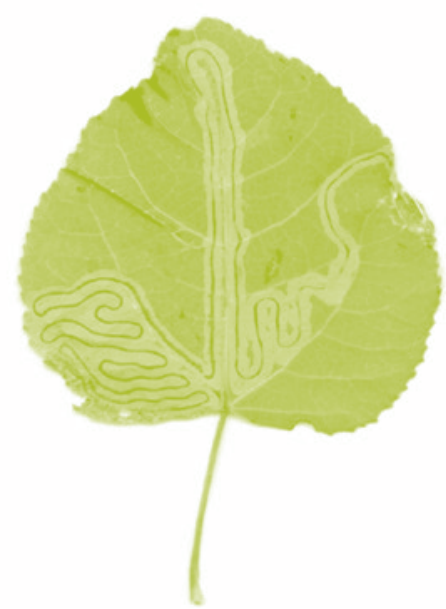

Figura 2: Una hoja con un patrón de túneles creados por un minador.

Desde una perspectiva más general, las líneas de guía de la figura 1 proporcionan a la tortuga la información acerca de su posición dentro de su entorno. Las señales posicionales y direccionales tienen un impacto fundamental en el comportamiento animal, que van desde el mantenimiento de la distancia adecuada de la costa por muchos organismos que viven en los litorales sobre la orientación de los insectos y las aves con respecto al sol, y el uso de puntos de referencia por los animales que se dirigen hacia una meta conocida. Aunque estas conductas no suelen dejar huellas visibles, sin embargo, pueden ser conceptualizados como rutas en el espacio, y se interpretan como patrones. 
El uso de las señales ambientales es también un factor omnipresente en desarrollo de la planta. Por ejemplo, el desarrollo de las plantas trepadoras se guía por la geometría de su apoyo.

ii) Identificación de Patrones en Ecuación de Reacción-Difusión

Primero nos referimos a fenómenos que corresponden al siguiente modelo

$$
\partial_{t} u=\partial_{x}^{2} u+F(u) \operatorname{con} F(u)=\epsilon u-g u^{3}-h u^{5}
$$

donde $g= \pm 1$ y asumimos que existen soluciones del tipo onda viajera como la siguiente,

$$
\xi=x-c t, \text { para } c>0
$$

lo cual hace que la ecuación (1) se convierte en la siguiente ecuación

$$
d_{\xi}^{2} u+\xi d_{\xi} u+F(u)=0
$$

con dos soluciones asintoticas

$$
u(\xi \rightarrow-\infty)=u_{s} \quad \text { y } \quad u(\xi \rightarrow+\infty)=u_{0} \quad ;
$$

se observa que $u=u_{s}$ se traslada hacia $u_{0}=0$, como se ilustra en la siguiente figura 3,

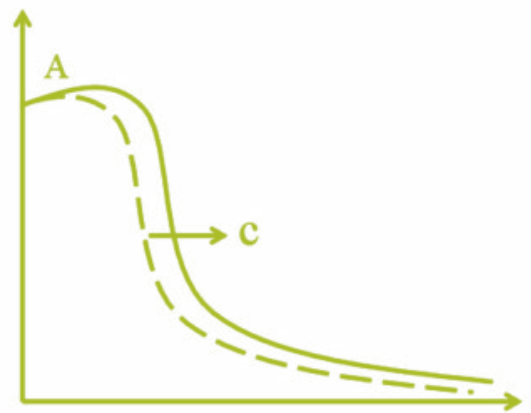

Figura 3: Ondas viajeras

En (1) si $g=+1$, corresponde a una bifurcación supercrítica, si $g=-1$ corresponde a una bifurcación subcritica tal como se ilustra en las siguiente figura 4 ,
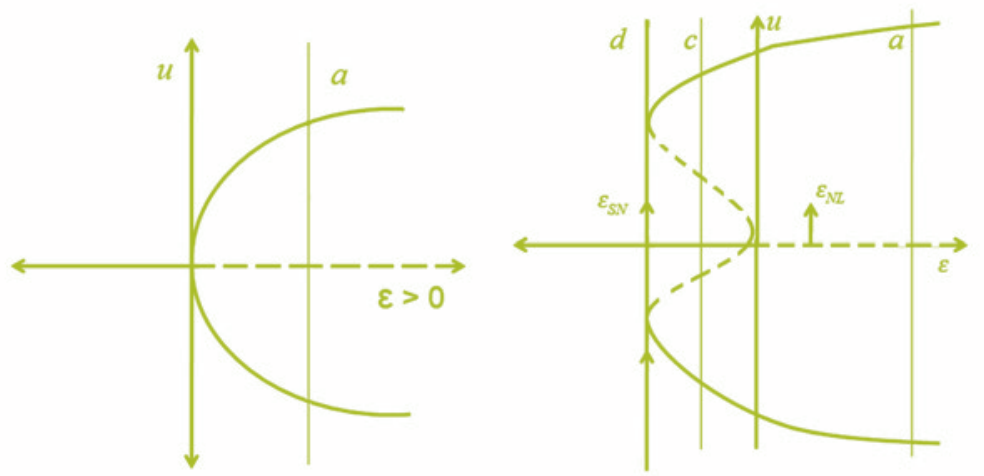

Figura 4: a) Bifurcación Supercrítica ; b) Bifurcación Subcrítica 
Para estudiar las bifurcaciones del sistema (1) en torno del parámetro $\epsilon=0$, establecemos la siguiente analogía:

$$
F(u) \longleftrightarrow \frac{d \phi}{d u}
$$

donde $\phi(u)$ es una función potencial y si además colocamos los cambios de variables $\xi \rightarrow T, u \rightarrow X$, obtenemos $\frac{d \phi}{d u}=\frac{d \phi(X(T))}{d X(T)}$.

En los dos casos, supercríticos y subcríticos la recta $\epsilon=a$, respectivamente, corresponde a los siguientes potenciales ilustrado en la figura 5 al pasar " $c$ " a través de $c=2 \sqrt{\epsilon}$.
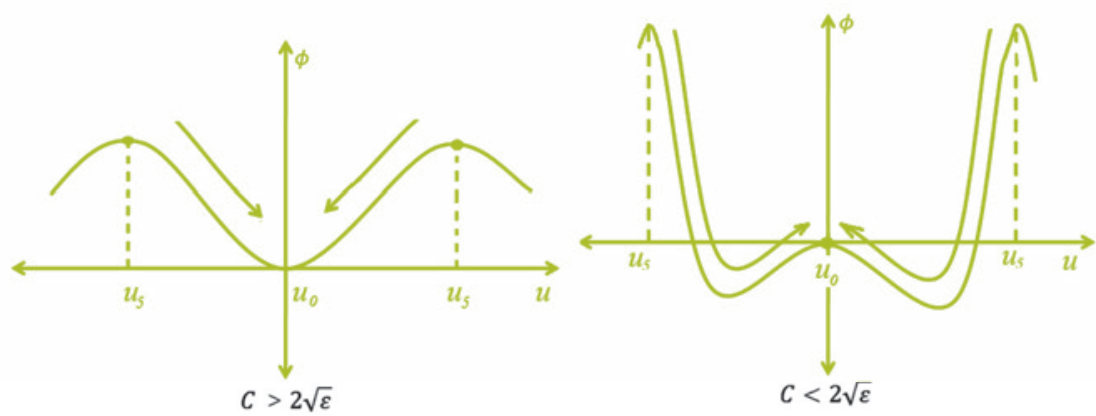

Figura 5: Potenciales para $\epsilon=a$ en los casos supercríticos y subcríticos

Los casos $\epsilon=d$ y $\epsilon=c$ para el caso subcrítico tiene respectivamente los siguientes potenciales ilustrado en la figura 6 ,

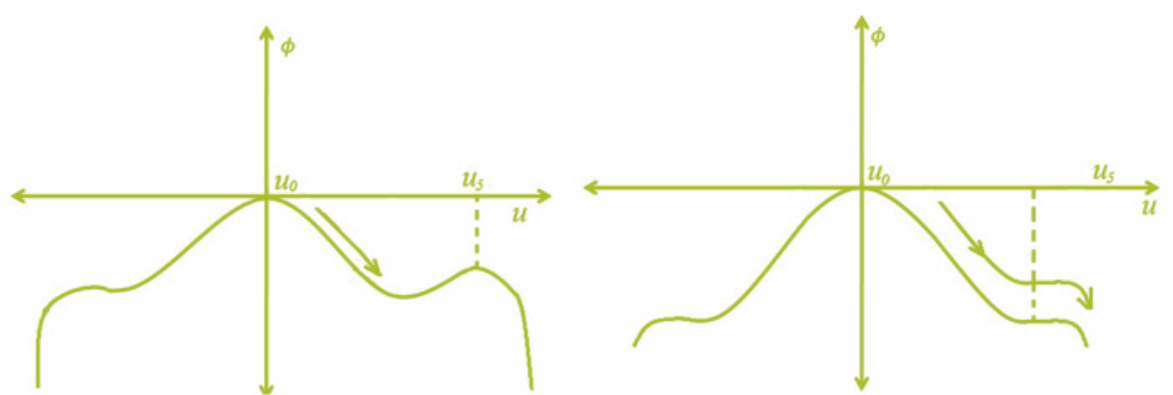

Figura 6: Potenciales para $\epsilon=d$ ó $\epsilon=c$ en los dos casos supercritico y subcritico

Así por ejemplo en el $\epsilon=a$, comenzando en el potencial máximo $u_{s}=u(\xi \rightarrow$ $-\infty$ ) hacia el potencial mínimo $0=u(\xi \rightarrow+\infty)$, según el signo de la velocidad de la onda "c" en $\xi=x-c t$.

En el caso subcrítico el valor $\epsilon=\epsilon_{S N}=d$ corresponde a una bifurcación silla-nodo, pues en el intervalo $\epsilon_{S N}<\epsilon<0$ hay un cambio de signo en el amortiguamiento.

iii) El Sistema de Turing es el siguiente,

$$
\partial_{t} u=F(u)+D \partial_{x}^{2} u \operatorname{con} D=\left(\begin{array}{cc}
D_{1} & 0 \\
0 & D_{2}
\end{array}\right), a_{i j}=\frac{\partial F_{i}}{\partial u_{j}}
$$

resulta de (4) que,

$$
A_{q}=A+D q^{2}
$$


con valores propios $\sigma_{q}$ dado por $A_{q} u_{q}=\sigma_{q} u_{q}$ con el vector propio $u_{p}=$ $u_{q} \mathrm{e}^{\sigma_{q} t} \mathrm{e}^{i q X}$ no nulo y $u_{q}$ es la configuración critica; además

$$
0=\operatorname{det}\left(A_{q}-\sigma_{q} I\right)=\sigma_{q}^{2}-\left(\operatorname{Traza}\left(A_{q}\right)\right) \sigma_{q}+\operatorname{det} A_{q}
$$

con las opciones mostradas en la siguiente figura 7 ,

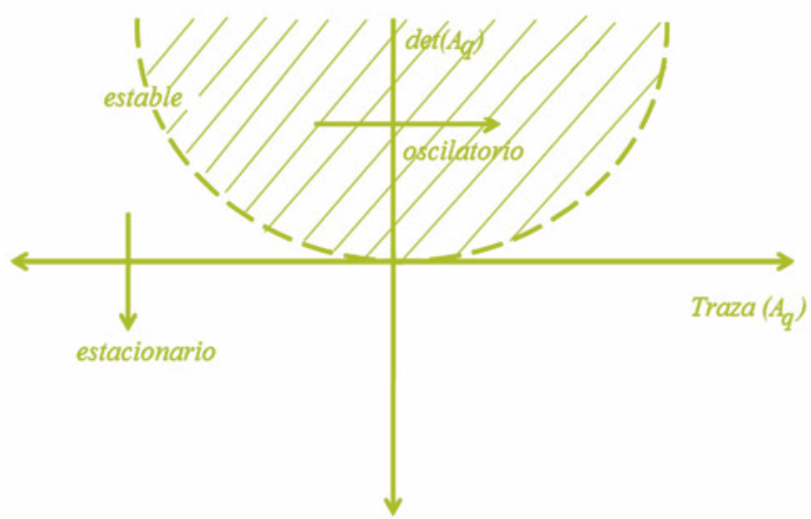

Figura 7: Gráficas para las bifurcación en un sistema de Turing

iv) El sistema de Turing bidimensional se describe mediante el siguiente sistema de ecuaciones,

$$
\left\{\begin{array}{c}
\frac{\partial C_{1}}{\partial t}=R_{1}\left(C_{1}, C_{2}\right)+D_{1} \nabla^{2} C_{1} \\
\frac{\partial C_{2}}{\partial t}=R_{2}\left(C_{1}, C_{2}\right)+D_{2} \nabla^{2} C_{2}
\end{array}\right.
$$

con estado de reposo homogéneo en $\left(\overline{C_{1}}, \overline{C_{2}}\right)$, esto es,

$$
\frac{\partial \overline{C_{1}}}{\partial t}=\frac{\partial \overline{C_{2}}}{\partial t}=\frac{\partial^{2} \overline{C_{1}}}{\partial x+\partial y}=\frac{\partial^{2} \overline{C_{2}}}{\partial x+\partial y}=0 \text {. }
$$

Así podemos linealizar (5) en torno a estos equilibrios homogéneos haciendo

$$
\begin{aligned}
& C_{1}^{\prime}(x, y ; t)=C_{1}(x, y ; t)-\overline{C_{1}} \\
& C_{2}^{\prime}(x, y ; t)=C_{1}(x, y ; t)-\overline{C_{2}}
\end{aligned}
$$

donde las soluciones de este sistema linealizado tienen la siguiente forma

$$
C_{i}^{\prime}(x, y ; t)=\alpha_{1} \mathrm{e}^{\sigma t} \cos \left(q_{1} x\right) \cos \left(q_{2} y\right)
$$

definidas en un rectángulo $L_{x} \times L_{y}$ con las condiciones de frontera adicionales siguientes,

$$
\left\{\begin{array}{c}
\frac{\partial C_{i}^{\prime}}{\partial x}=0 \text { en } x=0, L_{x} \\
\frac{\partial C_{i}^{\prime}}{\partial y}=0 \text { en } y=0, L_{y}
\end{array}\right.
$$

así obtenemos un conjunto discreto de números de ondas siguientes

$$
\begin{aligned}
q_{1} & =\frac{m \pi}{L_{x}}(m=0,1, \ldots) \\
q_{2} & =\frac{n \pi}{L_{y}}(n=0,1, \ldots) ;
\end{aligned}
$$


las configuraciones de los patrones se ilustran en el cuadro $L_{x} \times L_{y}$ proporcionando diversos patrones para parejas $(n, m)$ tal como se ilustran en la siguiente figura 8,
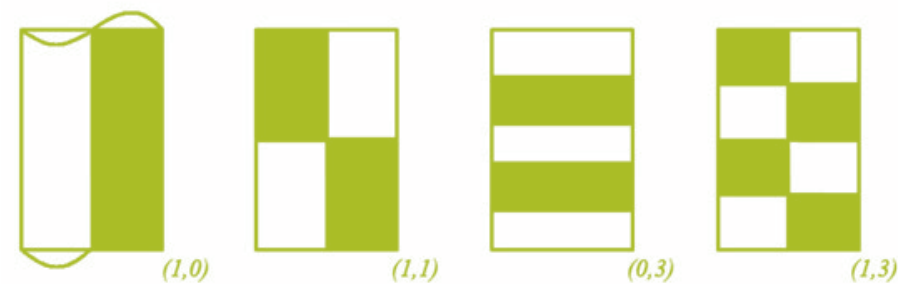

Figura 8: Identificación de patrones para un sistema de Turing bidimensional

v) El modelo de quimiotaxis de agregación celular corresponde al siguiente sistema no lineal:

$$
\left\{\begin{array}{l}
u_{t}=M u_{x x}-\left(u c_{x}\right)_{x} \\
c_{t}=D c_{x x}-c+g(u)
\end{array}\right.
$$

donde $u$ y $c$ son las concentraciones celular y del quimioatractor; en particular $g(u)=\frac{u}{1+u}$ con las siguientes condiciones de frontera

$$
0=u_{x}=c_{x} \text { en } x=0,1 \text { para } t>0 ;
$$

resultando el estado de equilibrio no trivial $\left(u_{0}, c_{0}\right)$ y relacionamos su estabilidad con las siguientes normas,

$$
h(u)=\max _{x \in[0,1]}\left|u_{x}\right| \quad y \quad N(u)=\int_{0}^{1} u(x) d x \quad ;
$$

las cuales tiene los siguientes significados: $N(\vec{u})$ es el número total de células presentes y se observa que $\frac{d}{d t} N(u(t, x))=0$ implica que $u$ y $c$ son espacialmente uniformes.

De la primera ecuación de este sistema (6) tenemos que

$$
u(x)=\lambda \mathrm{e}^{\frac{c(x)}{M}}
$$

donde $\lambda$ es una constante positiva porque $u \geqslant 0$, luego sustituyendo en la segunda ecuación del sistema (6) obtenemos

$$
D c^{\prime \prime}+G(c, \lambda)=0
$$

$\operatorname{con} G(c, \lambda)=g\left(\lambda \frac{c}{M}\right)+c$

De la ecuación (7) obtenemos el siguiente sistema bidimensional en el siguiente espacio de fases con variables $(c, p)$,

$$
\left\{\begin{array}{c}
c^{\prime}=p \\
p^{\prime}=-\frac{G(c, \lambda)}{D}
\end{array}\right.
$$

con las condiciones de frontera $p=0$ en $x=0,1$.

Por las condiciones de fronteras existen tres equilibrios $\left\{c_{1}, c_{2}, c_{3}\right\}$, dos sillas y un centro; resultando que el sistema (8) genera la siguiente configuración que se ilustra en la figura 9, 


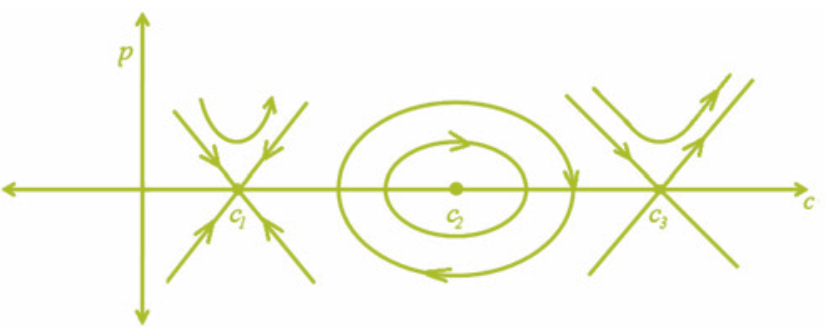

\section{Figura 9: Retrato de fases para el modelo de quimiotaxis}

vi) El modelo de Kuramoto-Sivashinsky corresponde a la siguiente ecuación con condiciones de frontera,

$$
\left\{\begin{array}{c}
u_{t}+u_{x x}+u_{x x x x}+\frac{1}{2}\left(u_{x}\right)^{2}=0 \\
0 \leq x \leq L, u(0, t)=u(L, t), u_{x}(0, t)=u_{x}(L, t) \quad ;
\end{array}\right.
$$

expresamos (9) en coordenadas de Fourier con respecto a " $x$ ", y obtenemos,

$$
\widehat{u_{t}}(k, t)=k^{2}\left(1-k^{2}\right) \widehat{u}(k, t),
$$

donde el número de ondas ' $q$ " con $|q|<1$ y $|q|=\frac{1}{\sqrt{2}}$ es el número de ondas del más rápido crecimiento lineal.

También representamos la solución $u(x, t)$ de la siguiente forma

$$
u(x, t)=\sum_{q \in \mathbb{Z}} a_{q}(t) \phi_{q}(x),
$$

es una combinación lineal de $\phi_{q}(x)=\exp \left(\frac{2 \pi i q x}{L}\right)$, soluciones linealmente independientes de (9), y cuyos coeficientes satisfacen la siguiente propiedad

$$
a_{-q}(t)=a_{q}^{*}(t),
$$

donde $a_{q}^{*}$ es el complejo conjugado de $a_{q}$ y además

$$
\left(\phi_{k}, \phi_{l}\right)=\int_{0}^{L} \phi_{k}(x) \phi_{l}^{*}(x)=\int_{0}^{L} \exp \left[\frac{2 \pi i(k-l) x}{L}\right]=\delta_{k l} \quad ;
$$

este producto interno es ortogonal en $L^{2}$, y además,

$$
\left|a_{0}(t)\right|=\int_{0}^{L}|u(x, t)| d x ;
$$

su tiempo promedio

$$
s(q)=\lim _{T \rightarrow \text { inf }} \frac{1}{T} \int_{0}^{T}|\widehat{u}(q, t)|^{2} d x
$$

De otro lado, para $l \in \mathbb{Z}$ en el espacio $L_{2}$ obtenemos el siguiente sistema,

$$
\left\{\begin{array}{c}
L\left[\dot{a}_{l}-\left(\frac{2 \pi l}{L}\right)^{2} a_{l}+\left(\frac{2 \pi l}{L}\right)^{4} a_{l}-\frac{1}{2}\left(\frac{2 \pi}{L}\right)^{2} \sum_{j \in \mathbb{Z}} j(l-j) a_{j} a_{l-j}\right]=0 \\
\int_{0}^{L} \exp \left[\frac{2 \pi i(x+j-l)}{L}\right] d x=0
\end{array}\right.
$$


así obtenemos el siguiente sistema $2 l+1$ espacio dimensional:

$$
\left\{\begin{array}{c}
\dot{a}_{l}=l^{2}\left[1-\left(\frac{2 \pi l}{L}\right)^{2}\right] a_{l}+\frac{1}{2} \sum_{j \in \mathbb{Z}} j(l-j) a_{j} a_{l-j} \\
\dot{a}_{0}=-\frac{1}{2} \sum_{j \in \mathbb{Z}} j^{2}\left(a_{j}\right)^{2} .
\end{array}\right.
$$

Para dar una idea de la estructura del sistema, se muestra las ecuaciones obtenidas por truncamiento en orden $k=4$, la cual resulta así:

$$
\left\{\begin{array}{c}
\dot{a}_{1}=\left[1-\left(\frac{2 \pi}{L}\right)^{2}\right] a_{1}-2 a_{1}^{*} a_{2}-6 a_{2}^{*} a_{3}-12 a_{3}^{*} a_{4} \\
\dot{a}_{2}=4\left[1-\left(\frac{4 \pi}{L}\right)^{2}\right] a_{2}-\frac{1}{2} a_{1}^{2}-3 a_{1}^{*} a_{3}-8 a_{2}^{*} a_{4} \\
\dot{a}_{3}=9\left[1-\left(\frac{6 \pi}{L}\right)^{2}\right] a_{3}-2 a_{1} a_{2}-4 a_{1}^{*} a_{4} \\
\dot{a}_{4}=16\left[1-\left(\frac{8 \pi}{L}\right)^{2}\right] a_{4}-2 a_{2}^{2}-3 a_{1} a_{3} ;
\end{array}\right.
$$

sistema que resulta ser invariante para el grupo $O(2)$ generado por las siguientes transformaciones $T_{\alpha}$ y $R_{l}$ :

$$
\left\{\begin{array}{c}
T_{\alpha}: a_{l} \longrightarrow \exp \left(\frac{2 \pi i l \alpha}{L}\right) a_{l} \\
R_{l}: a_{l} \longrightarrow a_{l}^{*}
\end{array}\right.
$$

La estabilidad en el equilibrio $a_{l}=0$ para todo $l$, puede ser determinada directamente mediante la siguiente linealización,

$$
\dot{a}_{l}=l^{2}\left[1-\left(\frac{2 \pi l}{L}\right)^{2}\right] a_{l}, l=1,2, \ldots
$$

La primera bifurcación ocurre cuando $u(x, t)=0$, en torno del cual realizamos su linealización

$$
\left\{\begin{array}{l}
\dot{x}_{l}=l^{2}\left[1-\left(\frac{2 \pi l}{L}\right)^{2}\right] x_{l} \\
\dot{y}_{l}=l^{2}\left[1-\left(\frac{2 \pi l}{L}\right)\right]^{2} y_{l},
\end{array}\right.
$$

con valor propio $\lambda_{l}=l^{2}\left[1-\left(\frac{2 \pi l}{L}\right)\right]^{2}$, el cual es de multiplicidad dos; por ello el origen es asintoticamente estable si $L<2 \pi$ e inestable si $L>2 \pi$ y esta es la primera bifurcación.

Su variedad central es tangente al subespacio generado $\left\{\cos \left(\frac{2 \pi l x}{L}\right), \sin \left(\frac{2 \pi l x}{L}\right)\right\}$, que resulta invariante para el grupo $O(2)$.

Para la segunda bifurcación, la cual corresponde a $L_{2}=4 \pi$, con valor propio $\mu=\left(\frac{2 \pi}{L_{2}}\right)-\left(\frac{2 \pi}{L}\right)^{2}$, tiene variedad central corresponde a la gráfica de $h_{l}$ 
siguiente $a_{l}=h_{l}\left(a_{1}, a_{1}^{*}, a_{2}, a_{2}^{*} ; \mu\right)$, con $l=3,4,5, \ldots$ en el siguiente sistema:

$$
\left\{\begin{array}{c}
\dot{a}_{l}=\left(\frac{3}{4}+\mu\right) a_{1}-2 a_{1}^{*} a_{2}-6 a_{2}^{*} h_{3}-12 h_{3}^{*} h_{4}-\sum_{k \geq 4} k(k+1) h_{k}^{*} h_{k+1} \\
\dot{a}_{2}=16 \mu a_{2}+\frac{1}{2} a_{1}^{2}-3 a_{1}^{*} h_{3}-8 a_{2}^{*} h_{4}-\sum_{k \geq 3} k(k+2) h_{k}^{*} h_{k+2}
\end{array}\right.
$$

colocando $L=2 \pi m(1-\mu)$ con $m \ll 1$, obtenemos la siguiente ecuación de bifurcación

$$
\dot{a}_{l}=16 \mu a_{2}-a_{1}^{2}-a_{2}\left(\frac{1}{2}\left|a_{1}\right|^{2}+\frac{1}{16}\left|a_{2}\right|^{2}\right) \quad ;
$$

la situación se repite y la variedad central es la gráfica $a_{l}=h_{l}\left(a_{m}, a_{m}^{*}\right)$, si $l \neq m$ ó $-m$ satisfaciendo $h_{-l}=h_{j}, a_{m}=h_{m}\left(\left|a_{m}\right|^{2}\right)$ en el siguiente sistema

$$
\dot{a}_{m}=m a_{m}\left(\mu-\frac{1}{12}\left|a_{m}\right|^{2}\right)+h_{m}\left(\left|a_{m}\right|^{2} ; \mu^{2}\right)+O\left(\left|a_{m}\right|^{4}, \mu, \ldots\right) \quad ;
$$

corresponde el siguiente sistema en coordenadas cartesianas reales,

$$
\left(\begin{array}{l}
\dot{x}_{m} \\
\dot{y}_{m}
\end{array}\right)=m^{2}\left[\mu-\frac{1}{2}\left(x_{m}^{2}+y_{m}^{2}\right)\right]\left(\begin{array}{l}
x_{m} \\
y_{m}
\end{array}\right)+\ldots,
$$

el cual también resulta $O(2)$ invariante, es una $O(2)$ ecuación de bifurcación del tipo de Pitckford.

Para $L=L_{m}$ la variedad invariantes dos-dimensional es el espacio tangente generado por $\left\{\cos \left(\frac{2 \pi l x}{L}\right), \sin \left(\frac{2 \pi l x}{L}\right)\right\}$ el cual produce un círculo de equilibrios para $L-L_{m}>0$.

vii) La ecuación de Swift - Hohenberg es la siguiente,

$$
\frac{\partial u}{\partial t}=\left[\sigma-\left(\nabla^{2}+1\right)^{2}\right] u-u^{3}
$$

para la cual buscamos soluciones de la forma

$$
u(x, y, t)=u_{0}(x+\phi(Y, T))+\varepsilon^{2} u_{1}(x, Y, T)+\varepsilon^{4} u_{2}(x, Y, T)+\ldots,
$$

donde $u_{0}$ es una solución del tipo de rayos o rollos, esto es solución espacialmente periódica con $T=\varepsilon^{2} t$ y $Y=\varepsilon y$; entonces (10) se convierte en,

$$
\varepsilon^{2} \frac{\partial u}{\partial T}=\sigma u-\left(\frac{\partial^{2}}{\partial x^{2}}+1\right)^{2} u-2 \varepsilon^{2} \frac{\partial^{2}}{\partial Y^{2}}\left(\frac{\partial^{2}}{\partial x^{2}}+1\right) u-\varepsilon^{4} \frac{\partial^{4} u}{\partial Y^{4}}-u^{3} ;
$$

sustituyendo (11) en (12) e igualando potencias de $\varepsilon$ hasta orden $O(1)$, obtenemos:

$$
\sigma u_{0}(x+\phi)-\left(\frac{\partial^{2}}{\partial x^{2}}+1\right)^{2} u_{0}(x+\phi)-u_{0}(x+\phi)^{3}=0,
$$

la cual no es útil porque $u_{0}(x)$ es estacionaria y $\phi(Y, T)$ no depende de $x$.

Hasta el orden $O\left(\varepsilon^{2}\right)$ tenemos

$$
\frac{d u_{0}}{d x} \frac{\partial \phi}{\partial T}=L_{0} u_{1}-2\left(\frac{d u_{0}}{d x}+\frac{d^{3} u_{0}}{d x^{3}}\right) \frac{\partial^{2} \phi}{\partial Y^{2}}-2\left(\frac{d^{2} u_{0}}{d x^{2}}+\frac{d^{4} u_{0}}{d x^{4}}\right)\left(\frac{\partial \phi}{\partial Y}\right)^{2}
$$


donde

$$
L_{0}=\sigma-\left(\frac{\partial^{2}}{\partial x^{2}}\right)-3 u_{0}^{2}
$$

donde $L_{0}\left(\frac{d u_{0}}{d x}\right)=\frac{\delta f}{\delta u} \frac{u_{0}}{d x}=0$, esto es $\frac{d u_{0}}{d x}$ esta en el núcleo de $L_{0}$; integramos (14) entre $-\frac{L}{2}$ y $\frac{L}{2}$ con respecto a $x$ con $L$ dado que $u_{0} x$ espacialmente periódico.

Hasta términos de orden $O\left(\varepsilon^{2}\right)$ la ecuación (14) es ahora

$$
L_{0}=2\left(\frac{d u_{0}}{d x}+\frac{d^{3} u_{0}}{d x^{3}}\right) \frac{\partial^{2} \phi}{\partial Y^{2}}+2\left(\frac{d^{2} u_{0}}{d x^{2}}+\frac{d^{4} u_{0}}{d x^{4}}\right)\left(\frac{\partial \phi}{\partial Y}\right)^{2},
$$

y del teorema de la alternativa de Fredholm tenemos:

$$
\int_{-\frac{L}{2}}^{\frac{L}{2}} \frac{d u_{0}}{d x}\left(\frac{d u_{0}}{d x}+\frac{d^{3} u_{0}}{d x^{3}}\right) \frac{\partial^{2} \phi}{\partial Y^{2}}+\frac{d u_{0}}{d x}\left(\frac{d^{2} u_{0}}{d x^{2}}+\frac{d^{4} u_{0}}{d x^{4}}\right)\left(\frac{\partial \phi}{\partial Y}\right)^{2} d x=0,
$$

Hasta orden $O(1)$ y cumple la condición de solubilidad. Análogamente se calcula el orden $O\left(\varepsilon^{4}\right)$, para obtener finalmente la siguiente ecuación de fases,

$$
\frac{\partial \phi}{\partial T}=D \frac{\partial^{2} \phi}{\partial Y^{2}}-K \frac{\partial^{2} \phi}{\partial Y^{4}}+g\left(\frac{\partial \phi}{\partial Y}\right)^{2} \frac{\partial^{2} \phi}{\partial Y^{2}}
$$

con $K$ y $g$ constantes reales.

viii) El modelo de las dos capas: Epidermis y Dermis

El sistema transformado puede ser escrita en términos de $x$ y $z$ mediante las siguientes ecuaciones,

$$
\left\{\begin{array}{c}
-\mu v\left(\frac{\partial^{3} \theta}{\partial z^{3}}+2 \frac{\partial^{3} \theta}{\partial z^{2} \partial x}+\frac{\partial^{3} \theta}{\partial z \partial x^{2}}\right)+\left(\frac{\partial^{2} \theta}{\partial z^{2}}+2 \frac{\partial^{2} \theta}{\partial z \partial x}+\frac{\partial^{2} \theta}{\partial x^{2}}\right) \\
-\beta\left(\frac{\partial^{4} \theta}{\partial z^{4}}+4 \frac{\partial^{4} \theta}{\partial z^{3} \partial x}+6 \frac{\partial^{4} \theta}{\partial z^{2} \partial x^{2}}+4 \frac{\partial^{4} \theta}{\partial z \partial x^{3}}+\frac{\partial^{4} \theta}{\partial x^{4}}\right) \\
+\tau\left(\frac{\partial^{2}}{\partial z^{2}}+\frac{\partial^{2}}{\partial z \partial x}+\frac{\partial^{2} \theta}{\partial x^{2}}\right)\left(\frac{n^{2}}{[1+v(1-\theta)]^{2}+c n^{2}}\right)=\rho \theta \\
-v \frac{\partial n}{\partial z}=D\left(\frac{\partial^{2} n}{\partial z^{2}}+2 \frac{\partial^{2} n}{\partial z \partial x}+\frac{\partial^{2} n}{\partial x^{2}}\right)+r n(1-n) \\
-\alpha\left(\frac{\partial}{\partial z}+\frac{\partial}{\partial x}\right)\left\{n\left(\frac{\partial}{\partial z}+\frac{\partial}{\partial x}\right)\left(\frac{1-\theta}{1+\gamma n}\right)\right\}
\end{array}\right.
$$

donde $\theta$ y $n$ son ahora funciones de $x$ y $z ; \theta(x, t)$ es la dilatación epitelial y $n(x, t)$ es la densidad de las celulas dermicas en la posición $x$ en el tiempo $t$, tal como se ilustra en la figura 10 , 


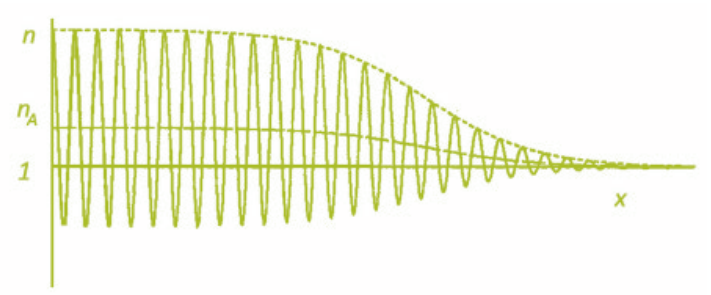

Figura 10: Una ilustración de las funciones de envolvente que representan cambios en la amplitud del patrón y en la densidad celular media

Representamos a estos por las curvas envolventes normalizadas $p(z), q(z)$, y $s(z)$, respectivamente. La última función $s(z)$ es, estrictamente hablando, no un envolvente, pero por conveniencia se describe como tal. Las siguientes propiedades se atribuyen a estas funciones envolventes:

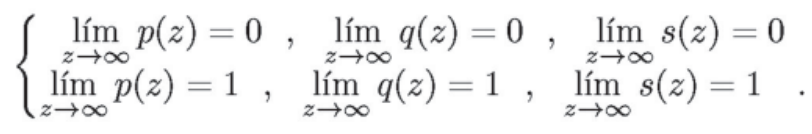

Asumimos además que la solución del sistema (18) se puede expresar como

$$
\left\{\begin{array}{c}
\theta(x, z)=p(z) \theta_{G}(x, z) \\
n(x, z)=q(z)\left[n_{G}(x, z)-n_{A}\right]+s(z)\left(n_{A}-1\right)+1
\end{array}\right.
$$

donde $\theta_{G}$ y $n_{G}$ son funciones que se determinarán más adelante. El valor de $n_{A}$ es la densidad media de células dérmicas cuando $z \rightarrow-\infty$, esto es,

$$
n_{A}=\lim _{z \rightarrow-\infty} \frac{1}{P} \int_{0}^{P} n(x, z) d x
$$

donde $P$ es el periodo del patrón de estado espacial constante en $z=-\infty$. La motivación para soluciónes de esta forma viene del examen de las soluciones ilustradas en la figura 10, en los que es evidente que las soluciones se puede aproximar muy de cerca por un patrón de longitud de onda espacial constante que tiene una amplitud que disminuye de forma monótona. Observe que la función envolvente $s(z)$ aparece en la expresión para $n(x, z)$. Esto es porque la densidad celular media, $n_{A}$, de la solución final no es necesariamente igual a la densidad celular inicial promedio $n=1$, que es el valor de estado estacionario, el cual es debido a la expresión de crecimiento logístico en nuestra ecuación dérmica conservación de células, así como el cambio en el patrón de desarrollo. De hecho, en la versión simplificada del modelo que se considerarán más adelante, el término crecimiento logístico se hace cero y desaparece este término en la envolvente.

Para determinar las funciones $n_{G}(x, z)$ y $\theta_{G}(x, z)$, se examina el comportamiento de la solución en el límite cuando $z \rightarrow \pm \infty$. Muy por detrás del borde de la perturbación, es decir, donde $z \rightarrow-\infty$, el sistema está en un estado estable espacialmente periódica, por ejemplo $S_{1}$. Representamos a este patrón de estado estacionario por $S_{1}=\left(\theta_{S_{1}}(x), n_{S_{1}}(x)\right)^{T}$, que es también la solución del problema no lineal $z$ - independiente. Así, a partir de (19) y (20) se deduce que

$$
\lim _{z \rightarrow-\infty}\left(\begin{array}{c}
\theta_{G}(x, z) \\
n_{G}(x, z)
\end{array}\right)=\left(\begin{array}{c}
\theta_{S_{1}}(x, z) \\
n_{S_{1}}(x, z)
\end{array}\right) \text {. }
$$


Muy por delante del borde de la perturbación, es decir, como $z \rightarrow \pm \infty$, el sistema está en el estado homogéneo estable $S_{0}$, donde $S_{0}=(0,1)$. A medida que la perturbación se propaga en la región homogénea, el estado de equilibrio es perturbado y una solución espacialmente periódico comienza a evolucionar. Nos representan la forma de este patrón inicial de la dilatación y la densidad celular por las funciones $\theta_{S_{0}}(x)$ y $\theta_{n_{0}}(x)$, respectivamente. En términos de las expresiones anteriores, (19) y (20), esto significa que

$$
\lim _{z \rightarrow-\infty}\left(\begin{array}{c}
\theta_{G}(x, z) \\
n_{G}(x, z)
\end{array}\right)=\left(\begin{array}{c}
\theta_{S_{0}}(x, z) \\
n_{S_{0}}(x, z)
\end{array}\right) .
$$

\section{Conceptos básicos en la identificación de patrones}

Para problemas sobre formación de patrones discretos generalmente tiene la siguiente forma analítica aproximada $u$ en torno del patrón critico $u_{0}$,

$$
u(x, t)=A(t) \sum_{q} \mathrm{e}^{i q x_{\perp}} . u_{0}\left(x_{\prime \prime}\right)+\text { c.c. }+\ldots
$$

en coordenadas de Fourier esta ecuación es

$$
\widehat{u}=\widehat{u_{0}} \mathrm{e}^{i q x+\sigma t}+c \cdot c
$$

En el caso de que el fenómeno es descrito por una ecuación diferencial parcial $\partial_{t} u=F\left(u, \partial_{x}\right)$, se dice que es espacialmente homogénea si $\frac{d u}{d t}=F(u, 0)$, con coordenadas de Fourier respectivamente

$$
\partial_{t} \widehat{u}(q)=D F\left(u_{c}, i q\right) \widehat{u}(q) \text { y } \frac{d}{d t} \widehat{u}(t)=\left(L(t)+i k c(t)+k^{2} D(t)+O\left(|q|^{3}\right) \widehat{u}(q)\right.
$$

Si $u_{c}$ es una solución critica, hacemos $u_{p}(x, t)=u(x, t)-u_{c}(x, t)$ y las variables espaciales $x_{\perp}$ son perpendiculares a $x_{l}$; la ecuación de amplitud lineal asociada con (21) se describe básicamente en terminos de la siguiente ecuación y parámetros,

$$
\begin{gathered}
d_{t} A=\sigma A \\
\epsilon=\frac{q-q_{0}}{q_{0}},
\end{gathered}
$$

donde $q_{0}$ es el número de ondas critico y el coeficiente de dispersión es $\sigma=$ $\tau^{-1} \epsilon+O\left(\epsilon^{2}\right)$, y $\tau$ es un rescalonamiento apropiado; y la ecuación de amplitud de onda no-lineal es

$$
d_{t} A=\sigma A+\partial_{x}^{2} A-\gamma|A|^{2} A+\ldots .
$$

En el caso particular de la ecuación real de Ginzburg - Landau tenemos,

$$
\frac{\partial A}{\partial T}=\sigma A-|A|^{2} A+\frac{\partial^{2} A}{\partial X^{2}},
$$


y escribiendo $A=R \mathrm{e}^{i \theta}$ obtenemos el siguiente sistema,

$$
\left\{\begin{array}{c}
\frac{\partial R}{\partial T}=\sigma R-R^{3}+\frac{\partial^{2} R}{\partial X^{2}}-R\left(\frac{\partial \theta}{\partial X}\right)^{2} \\
R \frac{\partial \theta}{\partial T}=2 \frac{\partial R}{\partial X} \frac{\partial \theta}{\partial X}+R \frac{\partial^{2} \theta}{\partial X^{2}} \quad ;
\end{array}\right.
$$

haciendo $\frac{\partial \theta}{\partial T}=0$ y multiplicando la segunda ecuación por $R$ se obtiene la siguiente ecuación,

$$
\frac{d}{d X}\left(R^{2} \frac{d \theta}{d X}\right)=0
$$

y así el momento angular constante se obtiene mediante la siguiente expresión,

$$
h=R^{2} \frac{d \theta}{d X} .
$$

El parámetro $\sigma(x)$ de la primera ecuación de (23) varia de positivo a negativo; en la región donde $\sigma(x)$ es negativo, solamente la solución nula es estable, así que $R=0$ y entonces $h=0$ y obtenemos las siguientes relaciones,

$$
\frac{d^{2} R}{d X^{2}}=-\frac{d V}{d R} \operatorname{con} \vee(R)=\frac{1}{2} \sigma R^{2}-\frac{1}{4} R^{2}+\frac{h^{2}}{2 R^{2}}
$$

y finalmente obtenemos,

$$
\frac{1}{2}\left(\frac{d R}{d X}\right)^{2}+V(R)=E
$$

donde $E$ es constante.

Si $\sigma(x)>0$ existen rollos estacionarios de la forma $A=R_{0} \mathrm{e}^{i q x} \operatorname{con} R_{0}^{2}=\sigma-q^{2}$, los cuales son como puntos fijos porque $R=R_{0}$ y $\frac{d R}{d X}=0$; para tales situaciones tenemos que $0<q^{2}<\sigma$ y entonces,

$$
h(q)=q\left(\sigma-q^{2}\right)
$$

cuya gráfica se muestra la siguiente figura 11 ,

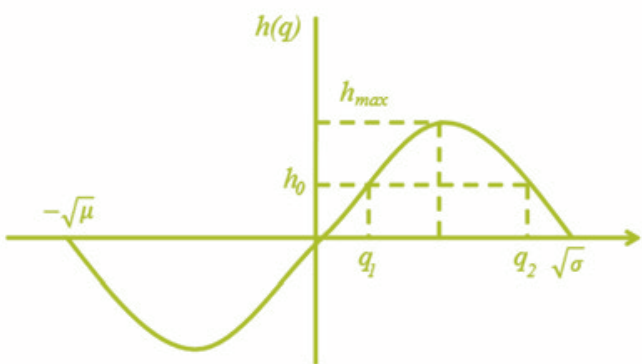

Figura 11: Gráfica de $h(q)$ 
tenemos que $h_{\max }=2\left(\frac{\sigma}{3}\right)$. Por ello obtenemos los siguientes comportamientos cualitativos para las soluciones estacionarias de la ecuación (21) los cuales se ilustran en los items $a$ ), b), c), d) de la figura 12 ,

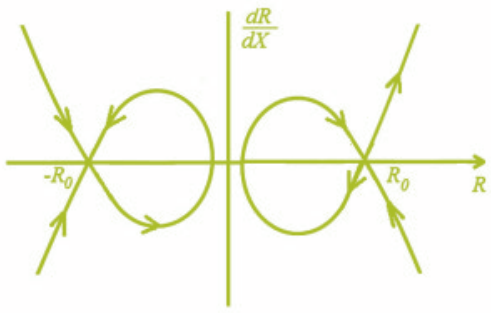

a) Si $\sigma>0,0<|h|<h_{\max }$

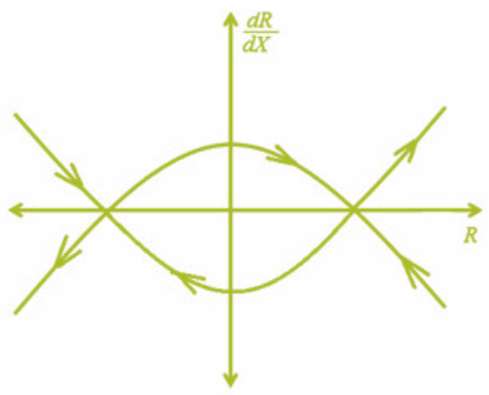

c) $\mathbf{S i} \sigma>0, h=0$

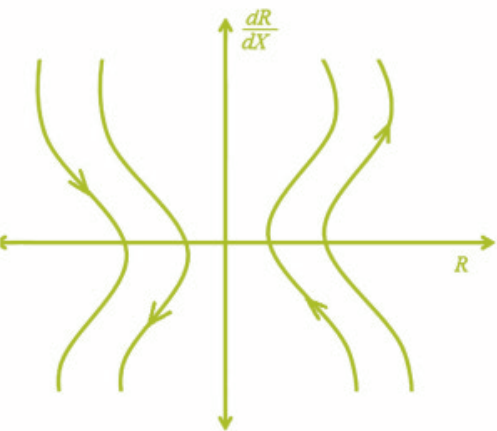

b) Si $\sigma<0,|h|>h_{\max }$

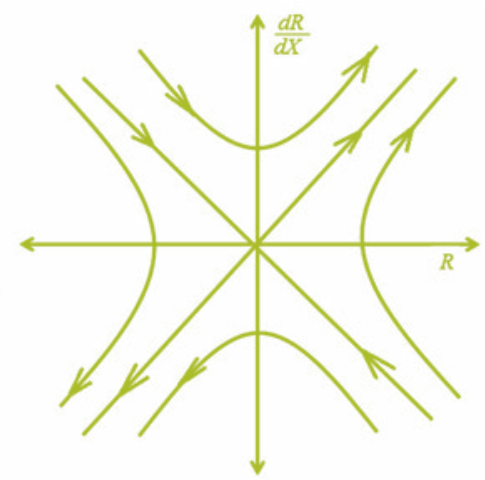

d) $\mathbf{S i} \sigma<0, h=0$

Figura 12: Dinámica de los equilibrios de (23) en los diferentes casos para " $\sigma$ " $\mathbf{y} " h "$

Si $h=0$ entonces $R \equiv 0$ y no hay formación de patrones, o $\frac{d \theta}{d R}=0$; así $\sin$ pérdida de generalidad podemos asumir $A=R$ real satisfaciendo

$$
0 \equiv \sigma R-R^{3}+\frac{d^{2} R}{d X^{2}}
$$

en el caso $\sigma>0$ se tiene soluciones espacialmente uniforme $R= \pm \sqrt{\sigma}$ y soluciones por defecto $R= \pm \sqrt{\sigma} \tanh \left(\sqrt{\frac{\sigma}{2 X}}\right)$. Linealizando alrededor de $A=0$, se logra $0=\sigma R+\frac{d R^{2}}{d X^{2}}$ con soluciones $R=a \sin (\sqrt{\sigma X})+b \cos (\sqrt{\sigma X})$ correspondiendo a soluciones espacialmente periódicas para $u$.

En general, la ecuación de fases, lejos del umbral $q_{0}$ esta relacionada con la ecuación de amplitud mediante las siguientes relaciones,

$$
\left\{\begin{array}{c}
u\left(x_{\perp}, x_{\prime \prime}\right) \cong u_{q_{0}}\left(\phi\left(x_{\perp}, x_{\prime \prime}\right)\right) \\
A(t)=|A| e^{i \phi} \\
\nabla \phi\left(x_{\perp}, t\right)=q\left(x_{\perp}, t\right)
\end{array} .\right.
$$


Si existe soluciones del tipo ondas viajeras tenemos $\bar{A}=a(\xi) \mathrm{e}^{i k_{0} c T} \mathrm{e}^{i \phi(\xi)}$ con $\xi=x+t \cdot c$ y $c>0$, entonces en las variables

$$
\left\{\begin{array}{c}
a=|\bar{A}|, k=|\bar{A}|^{-1} \frac{d}{d \xi}(|\bar{A}|) \\
K=\frac{d \phi}{d \xi}
\end{array}\right.
$$

estudiamos la formación de patrones mediante la solución del sistema de ecuaciones diferenciales siguientes con respecto a $\frac{d}{d \xi}$,

$$
\left\{\begin{array}{c}
\dot{a}=k a, \\
\dot{k}=-c k-1-k^{3}+k^{2}+a \\
\dot{K}=-c\left(k-k^{0}\right)-2 k K
\end{array}\right.
$$

cuyos puntos de equilibrio puede ser estable o inestable con conexiones heteroclínicas.

En la mayoría de los casos la amplitud $A_{q}(X, T)$ admite una solución del tipo ondas viajeras en coordenadas $X, T$; lo cual se obtiene haciendo: $X=x\left(\xi_{0}\right)^{-\frac{1}{2}}$, $\left.T=\left(t\left(\tau_{0}\right)\right)^{-\frac{1}{2}}\right), A_{q}=A\left(g_{0}\right)^{\frac{1}{2}}$; para obtener la siguiente expresión

$$
A_{q}(X, T)=a_{q} \mathrm{e}^{i\left(q(X+\theta)-\Omega_{q} T\right)},
$$

y satisface a la siguiente ecuación de amplitud con coeficientes complejos,

$$
\tau_{0} \partial_{t} A=\epsilon\left(1+i c_{0}\right) A+\left(1+i c_{1}\right) \xi_{0}^{2} \nabla A-\left(1-i c_{3}\right) g_{0}|A|^{2} A
$$

La ecuación de fases es la siguiente ecuación de derivadas parciales

$$
\partial_{t} \phi=-\Omega_{q}+\alpha \nabla_{x}^{2} \phi-\beta\left(\nabla_{x} \phi\right)^{2}
$$

con $\alpha=1-c_{1} c_{3}, \beta=c_{1}+c_{3}$ donde $\Omega_{q}=-c_{3}$ y $\Omega_{q}$ satisface la siguiente relación

$$
\tau_{0} \Omega_{q}=-\epsilon c_{0}+c_{1} \xi_{0}^{2} q^{2}-c_{3} g_{0}\left|a_{q}\right|^{2} .
$$

Para el estudio de bifurcaciones en el plano $\left(q, \operatorname{Real}\left(\sigma_{q}\right)\right)$, reemplazamos $(29)$ en (30) y tenemos en cuenta las siguientes relaciones

$$
\left\{\begin{array}{c}
w_{q}=-\operatorname{Im}\left(\sigma_{p}\right) \\
s=\frac{\partial w_{q}}{\partial q}=2\left(c_{1}+c_{3}\right) q
\end{array}\right.
$$

donde " $s$ " resulta ser la velocidad del grupo de ondas; entonces con los reescalonamientos siguientes: $A=\epsilon^{\frac{1}{2}}, X=\epsilon^{\frac{1}{2}} x, T=\epsilon t, \xi=X_{s} X_{p}, \partial_{\xi}=\epsilon^{-\frac{1}{2}} \partial x$, $\partial_{T}=\epsilon^{-1}\left(\partial_{t}+s \partial_{x}\right)$ obtenemos una expresión en variable compleja que simplifica la anterior ecuación de amplificación (33), esta es,

$$
\tau_{0}\left(\partial_{t} A+s \partial_{x} A\right)=\epsilon\left(1+i c_{0}\right) A-\left(1-i c_{3}\right) g_{0}|A|^{2} A,
$$

y entonces explicitamente $\sigma(q)$ se obtiene en la siguiente ecuación,

$$
\sigma(q)=-i s q+\tau_{0}^{-1} \epsilon\left(1+i c_{0}\right) q-\tau_{0}^{-1} \xi_{0}^{2}\left(1+i c_{1}\right) q^{2},
$$


con el valor crítico,

$$
\operatorname{Real}\left(\sigma\left(q_{c}\right)\right)=\operatorname{Real}\left(\sigma\left(\frac{i s \tau_{0}}{2 \xi_{0}^{2}\left(1+i c_{1}\right)}\right)\right)=0
$$

Mediante la conceptualización anterior se obtiene los siguientes clases de inestabilidades básicas en la identificación de patrones, esto es, tipos de bifurcaciones:

i) Para números de ondas próximas del número de ondas cercanas al valor critico $q_{0}$, obtenemos la siguiente ecuación de bifurcación,

$$
\sigma_{q}=\frac{1}{\tau_{0}}\left[\xi-\xi_{0}^{2}\left(q-q_{0}\right)^{2}\right],
$$

lo cual se ilustra en la siguiente figura 13 ,

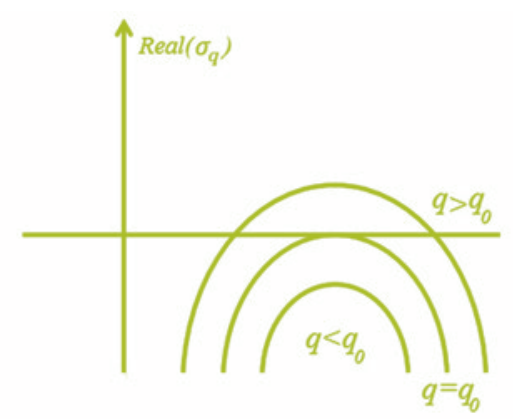

\section{Figura 13: Bifurcación del tipo i)}

ii) Para el caso de la simetría $q \longrightarrow-q$, correspondiente a patrones de la siguiente forma

$$
u(x, t)=A(t) \mathrm{e}^{i q x}+A^{*}(t) \mathrm{e}^{-i q x}
$$

genera la siguiente ecuación de bifurcación

$$
\operatorname{Real}\left(\sigma_{q}\right)=D\left(\xi q^{2}-\frac{1}{2} \xi_{0}^{2} q^{4}\right)
$$

cuya sucesión de gráficas se ilustran en la siguiente figura 14 .

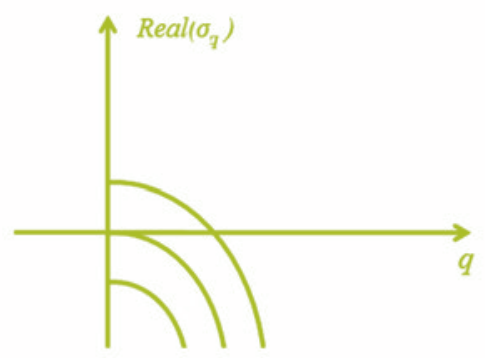

Figura 14: Bifurcación del tipo ii) simetría $q \longrightarrow-q$

iii) El caso oscilatorio, cuando " $q$ " atraviesa el eje vertical, se produce la siguiente ecuación de bifurcación:

$$
\operatorname{Real}\left(\sigma_{q}\right) \cong \frac{1}{\tau_{0}}\left[\xi-\xi_{0}^{2} q^{2}\right],
$$

tal como se ilustra en la siguiente sucesión de gráficas de la figura 15, 


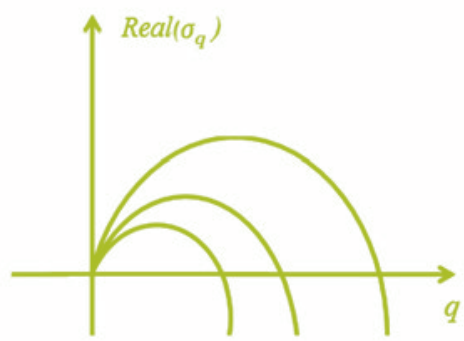

Figura 15: Bifurcación del tipo iii), esto es, del tipo oscilatorio

iv) Para el caso bidimensional continuo, esto es $q \in \mathbb{C}$, el reconocimiento de patrones $u(x, t)$ se identifica con la siguiente integral en variables complejas en la región que excluye al valor critico $q_{c}$,

$$
u(x, t) \approx \frac{1}{2 \pi} \int_{-\infty}^{+\infty} \mathrm{e}^{[i q x+\sigma(q) t]} d q \int_{-\infty}^{+\infty} u_{0}\left(x^{\prime}\right) \mathrm{e}^{-i q x^{\prime}} d x^{\prime},
$$

en la dirección $x=v \cdot t$ tenemos

$$
u(v \cdot t, t)=\frac{1}{2 \pi} \int_{-\infty}^{+\infty} \mathrm{e}^{[i q x+\sigma(q) t]} d q \int_{-\infty}^{+\infty} u_{0}\left(x^{\prime}\right) \mathrm{e}^{-i q x^{\prime}} d x^{\prime},
$$

de donde $\frac{d}{d q}[i q v+\sigma(q)]=0$ implica $c=v=i \frac{d \sigma}{d q}$ con $\sigma(q)=\epsilon-q^{2}$ y por ello $c=i \frac{\epsilon-q_{s}^{2}}{q_{s}}$, con velocidad crítica $c=2 \sqrt{\epsilon}$ pasando de una oscilación subamortiguada $c<2 \sqrt{\epsilon}$ a una oscilación sobreamortiguada en el caso $c>2 \sqrt{\epsilon}$;

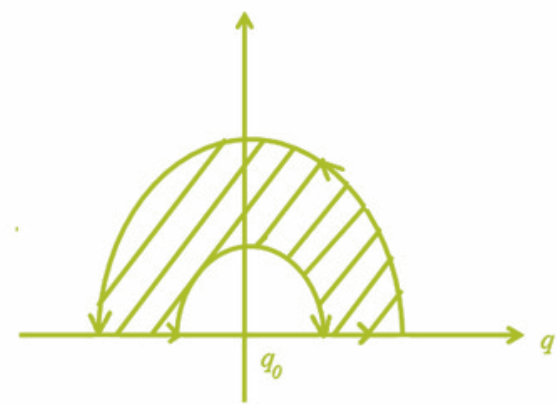

Figura 16: Región de integración para el caso bidimensional continuo el número de ondas bidimensional corresponde a

$$
\begin{gathered}
q=\left(q_{c}+q_{x}\right) \bar{x}+q_{y} \bar{y} ; \\
\mathrm{A}=a \mathrm{e}^{i\left[q_{x}(x+\theta)+q_{y}(y+\theta)\right]} \mathrm{e}^{i \sigma_{q} t} .
\end{gathered}
$$

Ecuación de fases es la siguiente

$$
\partial_{t} \phi=\left(\frac{1-3 q^{2}}{1-q^{2}}\right) \partial_{x}^{2} \phi+k \partial_{y}^{2} \phi \quad ;
$$

y debido a que la ecuación de amplitud es invariante para la siguiente transformación

$$
A \longrightarrow A \exp \left[i\left(\Delta y-\frac{\Delta^{2}}{2 q_{0}} x\right)\right],
$$


le corresponde a la siguiente ecuación de amplitud

$$
\tau_{0} \partial_{t} A=\epsilon+\xi_{0}^{2}\left(\partial_{x}-\frac{i}{2 q_{0}} \partial_{y}^{2}\right) q^{2}+g_{1}|A|^{2} A,
$$

entonces la siguiente ecuación de dispersión se obtiene sustituyendo A en (38),

$$
\sigma(q)=\tau_{0}^{-1} \xi_{0}^{2}\left(q-q_{0}\right)^{2}
$$

v) Estabilidad Eckhaus.

Observamos que el patrón de rollo ocurre, por ejemplo en el fenómeno convectivo de Rayleigh - Bénard - Layer; el cual explica cambios climáticos a cierta altura de la atmósfera y cuya representación es la dada mediante la figura 17.

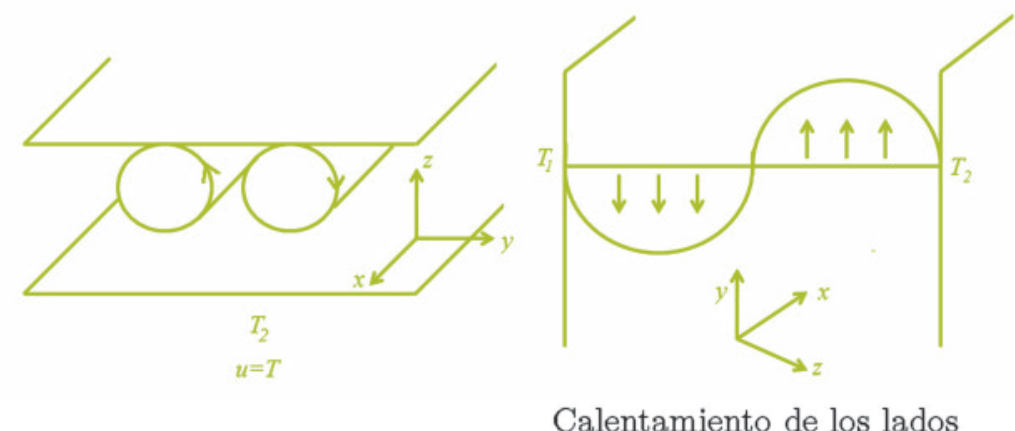

Figura 17: Patrón tipo "rollo" en el modelo Rayleigh - Bénard - Layer

Especialmente consideremos patrones del tipo rollos o rayos dados por soluciones de la forma

$$
u(X, Y ; t)=A(X, Y, T) \mathrm{e}^{i x}+c \cdot c+h \cdot o \cdot t
$$

donde la envolvente $A$ evoluciona de acuerdo a la siguiente ecuación de NewellWhitehead-Segel,

$$
\frac{\partial A}{\partial T}=\sigma A-|A|^{2} A+\left(\frac{\partial}{\partial X}-\frac{i}{2} \frac{\partial^{2}}{\partial Y^{2}}\right) A
$$

con $-\infty<X<\infty,-\infty<Y<\infty$; anulando el posible efecto en la frontera, consideremos el patrón del tipo rollo con envolvente dado por

$$
A=R_{0} \mathrm{e}^{i q x}
$$

Remplazando $A$ en (40) obtenemos

$$
\left\{\begin{array}{c}
R_{0}=\sigma-q^{2} \\
u=R_{0} \mathrm{e}^{i(1+\epsilon q) x}
\end{array}\right.
$$

y para estudiar la estabilidad del patrón consideramos la perturbación

$$
A=R_{0}(1-r) \mathrm{e}^{i(q x+\phi)}
$$

$\operatorname{con}|r|,|\phi| \ll 1$. 
La parte real e imaginaria al remplazar $A$ en la ecuación (40) proporcionan el siguiente sistema,

$$
\left\{\begin{array}{c}
\frac{\partial r}{\partial T}=-2 R_{0}^{2}-2 q \frac{\partial \phi}{\partial X}+\frac{\partial^{2} r}{\partial X^{2}}+\frac{\partial^{3} \phi}{\partial X \partial Y^{2}}+q \frac{\partial^{2} r}{\partial Y^{2}}-\frac{1}{4} \frac{\partial^{4} r}{\partial y^{4}} \\
\frac{\partial \phi}{\partial T}=2 q \frac{\partial r}{\partial X}+\frac{\partial^{2} \phi}{\partial X^{2}}-\frac{\partial^{3} r}{\partial X \partial Y^{2}}+q \frac{\partial^{2} \phi}{\partial Y^{2}}-\frac{1}{4} \frac{\partial^{4} \phi}{\partial y^{4}}
\end{array}\right.
$$

$\mathrm{El}$ efecto sobre la longitud de onda, donde las derivadas de la perturbación son pequeñas en comparación con las variables mismas, y tomando modos $r=\widehat{r} \mathrm{e}^{\sigma T+i k X+i l Y}, \phi=\widehat{\phi} \mathrm{e}^{\sigma T+i k X+i l Y}$ con $|l| \ll 1, k \sim l^{2}$ constantes reales, encontramos que:

$$
\left\{\begin{array}{c}
\sigma \widehat{r}=-2 R_{0}^{2} \widehat{r}-2 i q k \widehat{\phi}-k^{2} \widehat{r}-i k l^{2} \widehat{\phi}-q l^{2} \widehat{r}-\frac{l^{2}}{4} \widehat{r}, \\
\sigma \widehat{\phi}=2 i q k \widehat{r}-k^{2} \widehat{\phi}+i k l^{2} \widehat{r}-q l^{2} \widehat{\phi}-\frac{l^{2}}{4} \widehat{\phi} .
\end{array}\right.
$$

Eliminando $\widehat{r}$ y $\widehat{\phi}$ resulta una ecuación cuadrática en $\sigma$ como la siguiente,

$$
\left(\sigma+2 R_{0}^{2}+k^{2}+q l^{2}+\frac{l^{4}}{4}\right) \cdot\left(\sigma+k^{2}+q l^{2}+\frac{l^{4}}{4}\right)=k^{2}\left(2 q+l^{2}\right)^{2},
$$

lo cual tiene las siguientes relaciones dos raíces

$$
\left\{\begin{array}{c}
\sigma_{1}=-2 R_{0}^{2}+O(k) \\
\sigma_{2}=-k^{2}\left(1-\frac{2 q^{2}}{R_{0}^{2}}\right)-q l^{2}-\frac{l^{4}}{4}+O\left(k^{3}\right)
\end{array}\right.
$$

El primer valor propio es $\sigma_{1} \approx-2 R_{0}^{2}<0$ corresponde al autovector $(\widehat{r}, \widehat{\sigma})=$ $(1, O(k))$ y los rollos correspondientes son estables; el segundo valor propio $\sigma_{2}$ corresponde a una verdadera modulación porque contiene $q, k, l$ y estos no pueden aparecer en el patrón, su autovector es

$$
\left(\begin{array}{c}
O(K) \\
1
\end{array}\right)
$$

el cual describe la evolución lenta con escala de tiempo del orden $O\left(l^{-2}\right)=$ $O\left(k^{-1}\right)$; y reemplazando en la primera ecuación de (44) la perturbación de la fase $\phi$ mediante el cambio de escala

$$
\frac{\partial}{\partial X} \longrightarrow \delta \frac{\partial}{\partial X}, \frac{\partial}{\partial y} \longrightarrow \delta^{\frac{1}{2}} \frac{\partial}{\partial Y}, \frac{\partial}{\partial T} \longrightarrow \delta \frac{\partial}{\partial T}
$$

con $|\delta| \ll 1$ obtenemos,

$$
\left(2 R_{0}^{2}+\delta \frac{\partial}{\partial T}-\delta q \frac{\partial^{2}}{\partial Y^{2}}+O\left(\delta^{2}\right)\right) r=\left(-2 \delta q \frac{\partial}{\partial X}+O\left(\partial^{2}\right)\right) \phi ;
$$

luego multiplicando en ambos lados por 


$$
\left(2 R_{0}^{2}-\delta \frac{\partial}{\partial T}+\delta q \frac{\partial^{2}}{\partial Y^{2}}\right)
$$

obtenemos

$$
r=-\frac{\partial q}{R_{0}^{2}} \frac{\partial \phi}{\partial X}+O\left(\delta^{2}\right)
$$

Para perturbaciones en la dirección transversal $X$, donde $l=0$, reemplazamos en la segunda ecuación de (44) y obtenemos

$$
\sigma_{2}=-k^{2}\left(1-\frac{2 q^{2}}{R_{0}^{2}}\right)+O\left(k^{2}\right),
$$

con valor propio positivo para rollos en la región $R_{0}^{2}<2 q^{2}\left(\sigma<3 q^{2}\right)$. De esta forma se describe la conocida inestabilidad de Eckhaus.

\section{Avances sobre las estructuras localizadas en formación de patrones}

\section{Conclusión}

En este artículo hemos presentamos una metodología para describir las bifurcaciones que generan los sistemas dinámicos de la identificación de patrones que surgen en fen'omenos de origen interdisciplinarios; además analizamos la cualidad de los equilibrios inestables, lo cual es útil para retroalimentar el conocimiento de dichos fenómenos.

En particular hacemos una descripción de las bifurcaciones del coeficiente de dispersión " $\sigma$ " como función del número de ondas "q", dado que estos parámetros son los que caracterizan la identificación de patrones en los casos particulares y son relevantes en modelos no-lineales.

\section{Referencias bibliográficas}

1. Grindrod, Peter. The Theory and applications on Reaction-Difussion Equations, Patterns a Waver, Oxford, 1996.

2. Golubitsky, Martin; Dan Luss and Steven Strogatz. Pattern Formation Continuos and Compled Systems, Springer. 1999.

3. Dollmanam A. and A. van Harter. Nonlinear dynamics and pattern formation in the natural environment, Longman, 1995.

4. Holmes, Philip. Turfulence, Coherent Structures Dynamical Systems and Symmetry; Cambridge, Monographic on Mechanics, Cambridge, 1996.

5. Hoyle Rebecca. Pattern Formation, Cambridge, 2006. 
6. Cross Michael and Herny Greenside. Pattern Formation and Dynamics in nonequilibrium Systems; Cambridge, 2009.

7. Kuzretzov Yri A. Elements of applied Bifurcation Theory, Springer 2010.

8. Cantwell Brian J. Introduction to Symmetry Analysis, Cambridge, 2002.

9. Grindrod, Peter. On models of dispersion at Macroscopic Scales, Bath Institute for Complex Systems, preprust, 2006.

10. Murray J. D. Generation of Biological Pattern and form; IMA journal of Mathematics Applied in Medicine and Biology 1(1994):51-75.

11. Cowygen, Gerhard C. y otros; An envelope Methord for analyzing secuencial pattern formation, SIAM J. Applic Mathematic; 61(1)213,231. 